\title{
L'importance De La Dimension Éthique Dans La Performance Et La Gouvernance Des Organisations Publiques : L'apport De L'institutionnalisation Des Pratiques Éthiques Dans Le Secteur Public Marocain
}

\author{
Akhlaffou Mohamed \\ El Wazani Youssef \\ Souaf Malika
}

Equipe de Recherche en Management et Performance des Organisations Publiques, Privées et de l'Economie Sociale, Ecole Nationale de Commerce et de Gestion, Université Ibn Zohr, Agadir, Maroc

\section{doi: 10.19044/esj.2016.v13n2p100 URL:http://dx.doi.org/10.19044/esj.2016.v13n2p100}

\begin{abstract}
The aim of this paper is to analyse the positioning of ethical dimension in the New Public Management (NPM), and the influence of the institutionalization of ethical practices on governance and performance of public and non-profit organizations. The objective is then, to show how the theory of accountability and NPM incorporate the ethical factors, and the place given to ethics in the construction process of public action, the sensemaking in public organizations and its relationship with the position of ethics in the public management. This will be done through a case study on the ethical dimension in the management Morocco public organizations, by proposing recommendations to make their management mode more ethical, transparent and efficient.
\end{abstract}

Keywords: Ethical practices, New Public Management, Public Performance, Governance, Moroccan Public Organizations, Accountability

\section{Résumé}

Dans le présent article, nous nous intéressons au positionnement de l'éthique dans le nouveau management public (NPM), et l'impact de l'intégration des pratiques éthiques sur la performance et la gouvernance des organisations publiques et des organisations à but non lucratif. L'objectif est donc, de montrer comment le NPM et la théorie de reddition des comptes intègrent-ils la dimension éthique, et dans quelle mesure ces deux théories 
prennent en considération l'éthique dans le processus de construction de l'action publique, puis le sens et le non sens dans le management public et ses liens avec la position de l'éthique dans la gestion des organisations publiques. Et tout cela, à travers une étude de cas sur la dimension éthique dans la gestion des organisations publiques au Maroc, en proposant ainsi des recommandations pour rendre leur style de gestion plus performant, plus transparent et plus éthique.

Mots-clés : Pratiques éthiques, Nouveau Management Public, Performance Publique, Gouvernance, Organisations Publique Marocaines, Reddition de comptes

\section{Introduction}

Face aux défis d'un marché mondialisé marqué par un certain nombre de dilemmes moraux qui peuvent entrainer des dysfonctionnements dans les cycles de vie des organisations, il existe un marché éthique dans le cadre duquel, les managers et les entrepreneurs adoptent une vision partagée qui combine la responsabilité économique et la responsabilité morale, afin d'améliorer l'image de marque de leurs organisations, leur performances et leur compétitivité, et notamment dans la gestion publique.

En effet, l'amélioration de la gestion publique passe nécessairement par une conduite dictée par le respect des règles éthiques; puisque les normes réglementaires demeurent insuffisantes pour encadrer l'action des agents publics.

Les travaux sur les organisations publiques et les organisations à but non lucratif, constatent des problèmes managériaux dus à l'absence de sens dans leur gestion (AKHLAFFOU, EL WAZANI et SOUAF, 2016 ; Trosa et Bartoli, 2011). La mauvaise gestion interne des organisations publiques et la persistance de pratiques discutables sur le plan de l'efficacité et de l'efficience, sont autant d'handicaps nuisant à la mise en place d'un style de management performant et éthique. Par conséquent, des interrogations persistent quant aux apports des théories dans le domaine notamment le nouveau management public et la reddition des comptes.

Dès lors, nous nous posons plusieurs questions à savoir : comment le NPM et la théorie de reddition des comptes intègrent-elles la dimension éthique ? Ces deux théories prennent-elles en considération l'éthique dans le processus de construction de l'action publique? Quel est l'impact des pratiques éthiques sur la performance et la gouvernance des organisations publiques ? Le sens et le non sens dans le management public, est-il une configuration de la position de l'éthique dans la gestion des organisations publiques? 
Dans la présente étude, nous nous intéressons au positionnement de l'éthique dans la sphère publique marocaine et la pertinence du Nouveau Management Public (NPM) dans l'intégration de la dimension éthique dans la gestion des organisations publiques et des organisations à but non lucratif. L’objectif est donc, de montrer comment les pratiques éthiques impactentelles l'efficacité de la gestion de la chose publique au Maroc, et dans quelle mesure les deux théories précitées prennent en considération l'éthique dans le processus de construction de l'action publique, puis le sens et le non sens dans le management public et sa relation avec la position de l'éthique dans la gestion des organisations publiques.

\section{La gestion éthique dans le secteur public}

L’amélioration de la gestion publique passe nécessairement par une conduite dictée par le respect des règles éthiques; puisque les normes réglementaires demeurent insuffisantes pour encadrer l'action des agents publics.

En effet, les travaux sur les organisations publiques et les organisations à but non lucratif, constatent des problèmes managériaux dus à l'absence de sens dans leur gestion (Akhlaffou, El wazani et Souaf, 2016 ; Trosa et Bartoli, 2011). Dans ce qui suit, nous allons essayer d'exposer les principales caractéristiques du nouveau management public, ainsi que l'apport de la dimension éthique dans ce mode de gestion et dans le principe de reddition des comptes.

\section{Le nouveau management public (NMP)}

Hood définit le NMP de la manière suivante : « l'ensemble des doctrines administratives, sensiblement similaires, qui ont dominé le programme de réforme bureaucratique dans beaucoup de pays depuis la fin des années 70 » (Hood, 1991, 3-4). Le NMP est né au début des années 1980 au Royaume-Uni et en Nouvelle-Zélande, et s'est déployé ensuite dans la plupart des pays de l'OCDE. Le NMP est une nouvelle forme de gestion publique basée sur une culture du résultat, et qui est le résultat des réformes que connaît l'administration publique, pour répondre au problème de l'inefficacité des anciens modes de gestion dans le secteur public ; via une transposition des méthodes et mécanismes de management du secteur privé au secteur public.

En effet, les caractéristiques du NMP sont les suivantes :

- une séparation de la prise de décision stratégique relevant du pouvoir politique, de la gestion opérationnelle relevant de l'administration ;

- l'orientation des activités administratives et l'allocation des ressources en fonction des produits et services à délivrer plutôt qu'en fonction de règles ou procédures ; 
- la décentralisation et l'instauration d'agence comme instrument de régulation ;

- l'abandon du statut de fonctionnaire et l'avancement à l'ancienneté des agents pour une rémunération au mérite ;

- l'introduction des mécanismes de marché dans l'offre de biens et de services d'intérêt général ;

- la logique de la transparence tant sur la qualité que sur les coûts des prestations ;

- la recherche de l'efficience dans l'emploi des fonds publics ;

- la participation des usagers dans la définition et l'évaluation des prestations publiques.

\section{La dimension éthique dans le nouveau management public}

Victor et Cullen (1988), reconnus comme fondateurs de la théorie du climat éthique, définissent ce concept comme « l'ensemble des perceptions partagées de ce qui est un comportement éthiquement correct et de la manière dont les problèmes éthiques devraient être traités dans une organisation » (Victor et Cullen, 1988). Les deux auteurs attirent l'attention sur le fait que le climat éthique réunit l'ensemble des règles et des valeurs établies qui visent à déterminer ce qui est moralement mauvais ou bon, injuste ou juste dans un contexte organisationnel précis. Plus fraîchement, Arnaud (2010) définit ce construit de climat éthique comme «les perceptions globales et partagées des individus au sein d'une organisation reflétant le contenu et la force des valeurs éthiques, des normes, des attitudes, des sentiments et des comportements qui prévalent dans un système social».

A l'instar du secteur privé, le secteur public tente, depuis quelques années, d'introduire des principes et des règles éthiques de nature contraignante dans la gestion des administrations publiques. L'éthique dans la sphère publique, peut être définie selon Kernaghan (1993), comme un ensemble de règles et de principes de conduite correcte au sein d'une organisation publique.

Les agents publics ont un pouvoir discrétionnaire important lié aux différents aspects de la gestion publique, tels que la gestion des deniers publics, la contribution à l'élaboration des politiques publiques et le rapport avec les administrés (les usagers, les entreprises...etc.). Pour encadrer toutes ces attributions et éviter tout usage arbitraire de ce pouvoir, des normes éthiques sont d'une importance primordiale et leur application constitue un indicateur important de la bonne gouvernance et un signe de la qualité de la performance organisationnelle.

L'introduction de la notion du nouveau management public (NMP), produit des positions divergentes quant au respect des normes éthiques. En effet, certains chercheurs pensent que l'adoption du NMP peut encourager 
des comportements non éthiques à cause des marges de manœuvre et de l'autonomie dont sont dotés les fonctionnaires. D'autres estiment qu'au contraire, le NMP va lutter contre les comportements non éthiques et va augmenter la transparence et la lisibilité de l'action publique, à travers les mécanismes de la responsabilisation et la reddition de comptes.

Selon les rapports de l'OCDE, pour lutter contre les comportements non éthiques, il est nécessaire de renforcer les formations dans les domaines de l'éthique afin de sensibiliser les fonctionnaires à l'importance de respecter les valeurs éthiques. Dans le même sens, et pour maintenir l'intégrité et la moralité au sein des organismes publics, plusieurs mesures sont à prendre en considération. Nous en citons quelques unes :

- $\quad$ Respecter les délais fixés ;

- $\quad$ Donner des avis motivés pour les décisions prises ;

- $\quad$ Notifier les conflits d’intérêt ;

- $\quad$ Recourir aux réglementations et directives spécifiques ;

- $\quad$ Assurer une mobilité régulière du personnel ;

- $\quad$ Procéder à des contrôles stricts.

La figure suivante illustre les huit valeurs du service public, jugées essentielles selon le rapport de l'OCDE :

Figure $\mathrm{N}^{\circ} 1$ : Les huit valeurs essentielles du service public

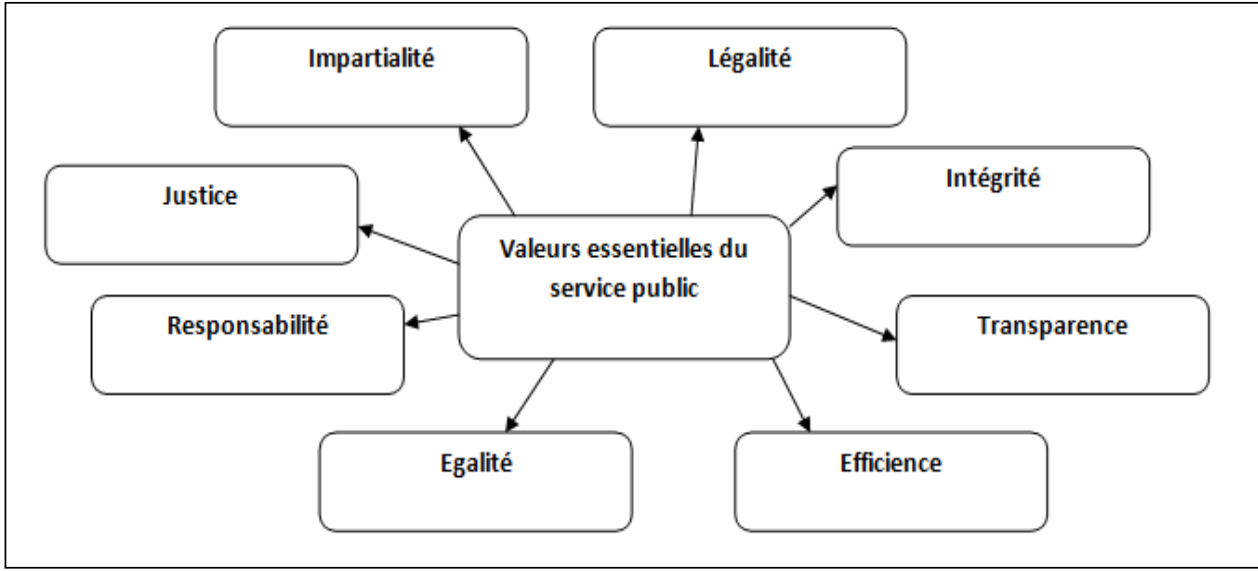

Source: Rapport OCDE (2000)

\section{La dimension éthique du principe de reddition des comptes}

La promotion de la gouvernance publique passera nécessairement, par la généralisation du principe de reddition des comptes (accountability) dans l'ensemble de la sphère étatique. Ce principe signifie que le mandataire ou le fonctionnaire a la responsabilité de rendre des comptes sur son action. La majorité des mandataires publics perçoivent encore leur fonction comme une récompense plutôt qu'une charge à assumer. C'est pour cela qu'il est 
nécessaire d’intégrer la dimension éthique dans la théorie de reddition des comptes.

Les administrations publiques se trouvent dans l'obligation de s’imposer une reddition des comptes exhaustive et transparente. Une telle reddition des comptes doit montrer comment l'administration publique s'est acquittée de sa mission et ainsi prouver que les fonds publics ont été utilisés avec efficience et efficacité, pour témoigner de leur performance. Celle-ci constitue une obligation de transparence du collaborateur envers son supérieur par rapport au travail accompli. Cette reddition des comptes peut être continue et concrétisée par un suivi régulier ou ponctuel via une évaluation permanente. Selon G. Clotuche $(1995)^{7}$, cette reddition des comptes peut être suivie de récompenses ou de sanctions, afin d'encourager le comportement souhaité.

Le principe de reddition des comptes ne serait utile que s'il est orienté par un système de valeur bien adapté ; car ce principe ne doit pas se fonder uniquement sur un ensemble de règles de natures juridique et technique. En effet, dans un contexte d'autonomie et de responsabilisation, il s’avère primordial que le fonctionnaire réfléchisse à la mission de son organisation, aux fins poursuivies et aux valeurs qu'elle porte.

C'est dans ce sens que l'éthique doit occuper une place importante dans la gestion de la chose publique. A cet effet, le mandataire doit agir selon l'esprit des normes éthiques qui lui sont applicables, et non selon une logique d'évitement des sanctions.

Dans le même sens, l'affirmation des valeurs partagées ${ }^{8}$ par les membres du personnel de l'administration publique (charte d'éthique, code déontologique, ...), tant à l'interne qu'à l'égard des citoyens qu'ils servent, parait essentielle pour rappeler les principes sur lesquels celles-ci s'appuient et pas uniquement pour les ajouter aux règles juridiques existantes.

\section{La gestion éthique dans l’administration publique marocaine}

Au Maroc, au cours de la dernière décennie, plusieurs efforts ont été entrepris pour renforcer l'intégrité des fonctionnaires et encourager les comportements éthiques au sein des établissements publics. Cela s’est fait notamment à travers la transposition des outils du secteur privé vers le secteur public et l'adoption de nouvelles stratégies pour lutter contre la corruption administrative. A titre d'exemple, nous citons la stratégie nationale de lutte contre la corruption qui a été adoptée fin 2015.

\footnotetext{
${ }^{7}$ G. CLOTUCHE, « La responsabilité des acteurs de la sécurité sociale », in Association internationale de la sécurité sociale, «La responsabilisation des acteurs de la sécurité sociale », Série européenne, 1995, Documentation de la sécurité sociale, n² 24, pp. 25-45.

${ }^{8}$ A savoir la compétence, l'impartialité, l’intégrité, la loyauté et le respect.
} 
La nouvelle constitution ${ }^{9}$ marocaine (2011) a consacré et pour la première fois, un chapitre entier à la bonne gouvernance ${ }^{10}$ (répondant aux exigences éthiques). Par conséquent, plusieurs principes fondamentaux ont été constitutionnalisés à savoir :

$>\quad$ Les services publics sont organisés sur la base de l'égal accès des citoyennes et citoyens, de la couverture équitable du territoire national et de la continuité des prestations ;

Ils sont soumis aux normes de qualité, de transparence, de reddition des comptes et de responsabilité, et sont régis par les principes et valeurs démocratiques consacrés par la Constitution ;

$>\quad$ Les agents publics exercent leurs fonctions selon les principes de respect de la loi, de neutralité, de transparence, de probité, et d'intérêt général. Ils assurent le suivi des observations, propositions et doléances des citoyens ;

$>\quad$ Ils rendent compte de la gestion des deniers publics conformément à la législation en vigueur et sont soumis, à cet égard, aux obligations de contrôle et d'évaluation ;

$>\quad$ Une charte des services publics fixe l'ensemble des règles de bonne gouvernance relatives au fonctionnement des administrations publiques, des régions et des autres collectivités territoriales et des organismes publics ;

Toute personne, élue ou désignée, exerçant une charge publique doit établir, conformément aux modalités fixées par la loi, une déclaration écrite des biens et actifs détenus par elle, directement ou indirectement, dès la prise de fonctions, en cours d'activité et à la cessation de celle-ci.

Les nouvelles technologies de l'information et de la communication (NTIC) ont également joué un rôle très important dans le renforcement des comportements éthiques. A ce stade, plusieurs projets ont été mis en place pour plus de transparence, de démocratie, de neutralité, et pour plus de proximité des services publics marocains aux citoyens. Nous pouvons citer à titre d'exemples :

- $\quad$ La dématérialisation des services douaniers (Système BADR) et la caisse nationale de la sécurité sociale (DAMNCOM)...etc.

- $\quad$ Nouvelles démarches pour la propriété industrielle et commerciale (OMPIC), automatisation de l'examen théorique pour l'obtention du permis de conduire, e-justice, @ujour,...

- $\quad$ Portail service-public.ma, gestion RH (GISRH), portail des marchés publics, portail CNOPS, ...

- $\quad$ Programme génie, CNIe, e-Impôts, ...

\footnotetext{
${ }^{9}$ Dahir n¹.11.91 du 27 chaabane 1432 (29 juillet 2011) portant promulgation du texte de la Constitution

${ }^{10}$ Chapitre 12 : articles 154 à 167.
} 


\section{La stratégie nationale de lutte contre la corruption}

Le Maroc a procédé, le 03 Mai 2016, au lancement officiel de la mise en œuvre de la stratégie nationale de lutte contre la corruption par la signature de conventions entre les organismes et secteurs concernés. Cette stratégie qui constitue la première du genre au Maroc, vise à améliorer l'intégrité et le climat éthique des affaires et le positionnement du Maroc à l'international, et à renforcer la confiance des citoyens envers les institutions publiques. Le pilotage de la stratégie de lutte contre la corruption est assuré par la commission nationale de lutte contre la corruption.

L'exécution de cette stratégie nécessitera une enveloppe budgétaire de 1,8 MMDH pour atteindre des objectifs ambitieux sur une durée de dix ans en trois étapes : (2016-2017), (2017-2020), et (2020-2025). Ces objectifs s'inscrivent dans un cadre contractuel de dix (10) programmes qui comprennent 239 projets. Il s'agit notamment des programmes suivants :

- $\quad$ Amélioration des services rendus aux citoyens et simplification des procédures, qui est coordonné par le Ministère de l’Intérieur ;

- $\quad$ Ethique des marchés publics, contrôle et reddition des comptes dont la coordination est confiée au Ministère de l'Economie et des Finances ;

- $\quad$ Ethique et déontologie, transparence et accès à l'information. Ce programme est chapeauté par le Ministère de la Fonction Publique et de la Modernisation de l'Administration ;

- Administration Electronique (dématérialisation des procédures et amélioration de la qualité de services) dont la coordination est assurée par le Ministère de l'Industrie, du Commerce, de l'Investissement et de l'Economie Numérique ;

- $\quad$ Renforcement des poursuites et de la répression, cordonné par le Ministère de la Justice et des Libertés ;

- $\quad$ Intégrité du monde des affaires et bonne gouvernance des entreprises. Ce programme est coordonné par la Confédération Générale des entreprises du Maroc (CGEM) ;

- $\quad$ Renforcement des capacités anti-corruption et introduction du cursus de l'intégrité et des formations en éthique dans l'enseignement. Cette mission est confiée au Ministre de l'Education Nationale et de la Formation Professionnelle ;

- Communication et sensibilisation dont la coordination est assurée par le Ministère de la Communication.

Il faut souligner dans ce cadre, que l'évaluation de cette stratégie est basée sur plusieurs indicateurs nationaux et internationaux. Il s'agit de l'indice de la perception de la corruption de transparency International, ainsi que les indicateurs du Doing Business et du World Economic Forum. A cet égard, l'ambition de cette stratégie, c'est d'améliorer, à l'horizon 2025, le classement du Maroc. L'objectif visé est d'obtenir une note de 60 sur 100 
pour l’indice de perception de la corruption. Le Maroc gagnerait, ainsi, 20 rangs dans le classement Doing Business de la Banque mondiale qui mesure la réglementation des affaires et son application effective, et remonterait enfin de 25 places dans l'indice de compétitivité, publié annuellement par le Forum économique mondial.

\section{Ethique et finances publiques au Maroc}

Les finances publiques constituent une branche de droit public, qui a pour objet l'étude des règles et des opérations relatives aux recettes et dépenses. Elles ont pour but l'étude des aspects juridiques, politiques et économiques des recettes et dépenses de l’Etat. Au Maroc, les finances publiques sont devenues un instrument majeur des politiques publiques, et un moyen vital aux mains des pouvoirs publics pour orienter et développer la vie économique et sociale.

La nouvelle Constitution du Maroc vise la consolidation de l'Etat de droit, la consécration des principes de séparation des pouvoirs, le renforcement de la bonne gouvernance et la corrélation entre la responsabilité et la reddition des comptes.

Il est donc nécessaire pour une bonne gouvernance financière publique, que la gestion des finances publiques, obéisse à des règles éthiques, basées sur des principes d'intégrité, de transparence, d’obligation de rendre compte, et d'équité. Par ailleurs, les normes réglementaires demeurent insuffisantes pour donner du sens et encadrer l'action des agents publics. Ceci explique cet engouement pour l'éthique qui apparaît désormais comme une valeur d'équilibre et de régulation. Elle puise en effet dans ce qu'il y a de meilleur dans la personne et ne se réduit pas à un catalogue de prescriptions uniformes (Sbai El Idrissi, 2007,75) ${ }^{11}$.

\section{Corruption et gouvernance financière des finances publiques}

La corruption est le détournement d'un mandat ou d'une position de pouvoir au profit d'intérêts privés. Elle permet d'assurer des avantages à ceux qui disposent de ressources de pouvoir, de statut, d'argent ou de protection.

Parmi les conséquences de la corruption, on peut citer la baisse des recettes publiques et l'aggravation des charges de l'Etat à cause du détournement de ressources, fuite de capitaux et/ou dissuasion de l'investissement, inégalité d'accès et détérioration des services publics et atteinte à la démocratie voire déstabilisation politique.

La formule suivante résume la corruption en quatre variables :

\footnotetext{
${ }^{11}$ El Idrissi, L. S. (2007). Intégrité, éthique et finances publiques au Maroc. Finance \& Bien Commun, (3), 74-80.
} 
$\mathbf{C}=\mathbf{M}+\mathbf{D}-(\mathbf{T}+\mathbf{A})$

$\mathrm{M}=$ Monopoles $; \mathbf{T}=$ Transparence et éthique

D = Pouvoirs discrétionnaires ; $\mathbf{A}=$ Accountability (Reddition des comptes)

C'est dans cet ordre d'idées qu'il faut renforcer le climat éthique, la transparence et la reddition des comptes au sein des établissements publics pour lutter contre ce fléau.

Une reddition des comptes éthique et transparente dans la gestion des finances publiques au Maroc

L'administration publique au Maroc doit s'imposer une reddition des comptes transparente, basée sur des normes éthiques et non seulement sur des règles techniques et juridiques. Le principe de reddition des comptes, pour porter ses fruits, doit bien sûr s'appuyer sur un système d'éthique bien fondé et sur des informations objectives et transparentes. Le seul témoignage des responsables évalués ne saurait suffire. A cet effet, ce principe doit démontrer que les fonds publics ont été utilisés avec efficience et efficacité.

Parallèlement, une reddition des comptes éthique exige au préalable quatre conditions en l'occurrence la sincérité des budgets, la clarté des opérations d'exécution, la sincérité des comptes et l'intégrité des mandataires.

Dès lors, il faut converger les efforts vers des programmes soutenant la moralité de l'action des mandataires qui gèrent les finances publiques, et encourager une auto-reddition des comptes, pour la rendre conforme aux obligations fixées. Cela exige une cohérence entre les convictions intimes et l'action publique et suppose la maîtrise de soi, l'intégrité et le courage moral nécessaires pour que l'exercice d'une activité soit compatible avec les convictions.

Pour une telle reddition éthique des comptes, nous proposons les recommandations suivantes, dans l'espoir que leur adoption contribuera à améliorer le climat éthique des organisations publiques Marocaines :

- Une promotion de la transparence et une obligation de rendre compte dans tous les nivaux de responsabilité ;

- Le renforcement des institutions de contrôle (Cour des comptes, ICPC, Conseil de la Concurrence...);

- Une éducation civique indispensable qui garantit le respect des valeurs éthiques ;

- La présentation des données comptables ainsi que des indicateurs (quantitatifs ou qualitatifs) qui mesurent l'efficience de l'administration dans l'utilisation des fonds publics ;

- L'accroissement de la lisibilité et de la transparence des procédures de contrôle interne au sein des organismes publics ; 
- L’amélioration de l'information financière (reddition des comptes, reporting lors de la préparation de la loi de finances et au cours de son exécution);

- La production et la publication de budgets spécifiques à l'attention des citoyens (faciles à comprendre) ;

- La mise en place de formations en gestion éthique au sein des organisations publiques et le respect des normes éthiques lors de la sélection des nouveaux recrus ;

- L’intégration de la dimension éthique dans le NMP en revisitant la théorie de reddition des comptes.

A partir de ce qui précède, on peut dire qu'il est nécessaire d'intégrer la dimension éthique dans la reddition des comptes, car les règles techniques et réglementaires demeurent insuffisantes pour encadrer l'action des agents publics. Dans le domaine des finances publiques comme dans d'autres domaines, cette dimension éthique parait donc devoir être établie par des autorités morales, puisque les finances publiques constituent un instrument de politique économique nécessaire à la stimulation de la croissance économique et au développement humain. Ainsi, il faut que l'éthique suscite spontanément l'adhésion, y compris dans des matières qui, telles les finances publiques, sont réputées techniques mais qui, en réalité, peuvent voir leurs principes essentiels ramenés à quelques normes éthiques, telles que la transparence et l'intégrité, avec une reddition des comptes éthique et complète.

\section{Conclusion}

La question des pratiques dites " éthiques », est entrée dans le champ de l'actualité depuis une dizaine d'années. La réflexion éthique en entreprise est au cœur des contradictions entre les logiques économiques et sociales. C'est une réflexion concernant la responsabilité de l'organisation vis-à-vis des acteurs internes et externes. Cette notion de responsabilité évoque l'obligation de justifier tout acte ou décision en fonction de normes et de valeurs morales, et de respecter les principes éthiques, tels que la responsabilisation et la reddition de comptes.

Dans ce sens, l'éthique englobe un ensemble des règles de conduite morale à suivre par tous les mandataires, pour une bonne gouvernance de l'action publique. A cet égard, la promotion de la gouvernance publique passera nécessairement, par la moralisation de l'action publique et la généralisation du principe de reddition des comptes (l'accountability) dans l'ensemble des échelles de la sphère étatique.

En effet, les travaux sur les organisations publiques et les organisations à but non lucratif, constatent des problèmes managériaux dus à 
l'absence de sens dans leur gestion (Akhlaffou, El wazani \& Souaf, 2016 ; Trosa et Bartoli, 2011).

Dans ce papier nous avons traité le positionnement de l'éthique et la reddition des comptes dans la gestion des organisations publiques et des organisations à but non lucratif au Maroc, et son impact sur la performance et la gouvernance de l'action publique.

L'amélioration de la qualité de la gestion publique passe inévitablement par le développement d'une nouvelle forme de reddition des comptes basée sur des règles éthiques. Cela suppose, l'introduction de techniques d’évaluation éthique dans les systèmes de décision, tout en assurant des contextes de transparence qui les mettent à l'abri des pressions du jeu politique ou administratif, susceptibles de les fausser. Cela suppose aussi une circulation des informations plus pertinentes assurant une communication optimale entre l'administration, le monde politique et les citoyens.

\section{References:}

1. AKHLAFFOU, M., EL WAZANI, Y., \& SOUAF, M. (2016). The Ethical Dimension in the New Public Management: Revisiting the Theory of Accountability, the Case of Public Finances in Morocco. European Scientific Journal, ESJ, 12(31).

2. Annexe au Code Marocain de Bonnes Pratiques de Gouvernance d'Entreprise (2008).

3. Antonmattéi, P., Vivien, P., \& LARCHER, M. G. (2007). Chartes d'éthique, alerte professionnelle et droit du travail français: état des lieux et perspectives. Droit social, 5, 522.

4. Arnaud A. (2010) Conceptualizing and Measuring Ethical Work Climate: Development and Validation of the Ethical Climate Index, Business \& Society, vol.49, $\mathrm{n}^{\circ} 2$, 345-358.

5. Bai, G. (2012). How Do Board Size and Occupational Background of Directors Influence Social Performance in For-profit and Non-profit Organizations? Evidence from California Hospitals.Journal of Business Ethics, 1-17.

6. Bartoli, A., Keramidas, O., \& Larat, F. (2012), Vers un management public éthique et performant. Revue française d'administration publique, (4), 629-639.

7. De Bry, F. (2008). Concept d'éthique l'éthique au cœur du management. Management \& Avenir, (6), 102-114.

8. Deslandes, G. (2012). Le management éthique. Dunod.

9. Eberle, D., Berens, G., \& Li, T. (2013). The Impact of Interactive Corporate Social Responsibility Communication on Corporate Reputation.Journal of Business Ethics, 1-16. 
10. El Idrissi, L. S. (2007). Intégrité, éthique et finances publiques au Maroc. Finance \& Bien Commun, (3), 74-80.

11. Eraly, A., \& Hindriks, J. (2007). Le principe de responsabilité dans la gestion publique. Reflets et perspectives de la vie économique, (1), 193-208.

12. Erhemjamts, O., Li, Q., \& Venkateswaran, A. (2012). Corporate Social Responsibility and Its Impact on Firms' Investment Policy, Organizational Structure, and Performance. Journal of Business Ethics, 1-18.

13. Göransson, M. (2010). La responsabilisation des hauts fonctionnaires aux différents niveaux de pouvoir. Courrier hebdomadaire du CRISP, (11), 7-83.

14. ICPC (2011), Bonne gouvernance entre situation actuelle et les dispositions de la nouvelle constitution de 2011, www.icpc.ma.

15. Kotler, P., \& Lee, N. (2008). Corporate social responsibility: Doing the most good for your company and your cause. Wiley. com.

16. Labelle, F. (2005). La performance sociétale organisationnelle comme convention sociale entre l'entreprise et son milieu: le cas d'Alcan au Saguenay-Lac-Saint-Jean (Doctoral dissertation, thèse de doctorat de gestion, UQAM, 2005. Retour).

17. Mazouz, B., Leclerc, J., \& Tardif, M. J. B. (2008). La Gestion Intégrée Par Résultats: Concevoir Et Gérer Autrement la Performance Dans L'administration Publique. PUQ .

18. Mercier, S. (1997). Une contribution à la formalisation de l'éthique dans les grandes entreprises, thèse de doctorat ès Sciences de Gestion, Université Paris IX Dauphine, septembre.

19. Michaud, Y. (2013). Qu'est-ce que le management responsable?: Confiance, décision, réflexivité. Eyrolles.

20. Moussé, J. (2001). Ethique des affaires: liberté, responsabilité: le décideur face à la question éthique (pp. 12958770-18152). Dunod.

21. Pitelis, C. N. (2013).Towards a More 'Ethically Correct'Governance for Economic Sustainability.Journal of Business Ethics, 1-11.

22. Trosa, S. \& Bartlo, A. (2011), Le management par le sens, au service du bien public, Collection profession cadre service public - ScérénCNDP, p : 246.

23. Van Doren, W., \& Lonti, Z. (2011). Contribution au débat sur la mesure de la performance de la gouvernance. Revue française d'administration publique, (3), 517-532.

24. Victor B., Cullen J. B. (1988) The organizational Bases of Ethical Work Climates, Administrative Science Quarterly, 33 (1), 101-125. 